\title{
La Capacidad de Innovación Tecnológica en la Universidad Autónoma del Estado de México
}

\author{
Antonio Arellano Hernández ${ }^{1}$ \\ Centro de Investigación y Estudios Avanzados en Ciencias \\ Agropecuarias, UAEM
}

La acción dirigida a separar la cultura y la técnica, entre el hombre y la máquina es falsa $y$ sin fundamento. Ella no recubre que ignorancia o resentimiento. Ella oculta un fácil humanismo y una realidad rica en esfuerzos humanos y fuerzas naturales y que constituye el mundo de los objetos técnicos, mediadores entre la naturaleza y el hombre.

Gilbert Simondon

\section{Introducción}

El presente trabajo aborda el tema de la capacidad de innovación tecnológica en la Universidad Autónoma del Estado de México como un ejercicio metodológico y teórico que pueda ser extensible a las condiciones de otras Universidades mexicanas.

Los problemas que están en el origen de este trabajo y que alimentan la reflexión y la búsqueda de elementos empíricos son los siguientes: ¿cuál es la característica del marco jurídico de la UAEM en relación a la investigación y la innovación tecnológica?, ¿cuál es la tendencia de investigación de la UAEM y cuál es el peso específico que tiene la investigación tecnológica?, ¿cuál es la experiencia de la UAEM en la vinculación Universidad-Sector productivo $^{2}$ para facilitar la congruencia entre demandas y ofertas de nuevos conocimientos y tecnologías? Todas estas cuestiones pueden ser resumidas en una sola, a saber: ¿Cuál es la capacidad de que la UAEM ofrezca a la sociedad innovaciones tecnológicas?

1 El autor agradece a la Coordinación General de Investigación y Estudios Avanzados de la UAEM las facilidades para contar con una parte de la información que sustenta este trabajo y al Mtro Jorge Arzate Salgada sus comentarios al presente artículo Asimismo reconoce la participación de Claudia Ortega Ponce en la elaboración de este trabajo mediante la recopilación y selección de información, así como en la revisión del mismo.

2 Frente a la gama de posibilidades que se utilizan para designar el sector al que se vincula la Universidad, nosotros hemos preferido utilizar el término Sector productivo para dejar ampliamente asentado que este sector puede ser el industrial, el empresarial, etc. 
En este trabajo se trata de ofrecer una respuesta tentativa a las tres primeras cuestiones mediante la ilustración y discusión de los elementos empíricos correspondientes. Tomando en consideración las tres respuestas parciales, se tratara de ofrecer una respuesta de carácter global sobre de la capacidad de la UAEM para innovar en tecnología.

No puede soslayarse la preocupación de la UAEM por profundizar y ampliar la investigación. Desde inicios de los años 60, la evolución de la investigación en la Universidad muestra un constante aumento en los efectivos de los investigadores, en las temáticas de investigación, en los convenios de intercambio con otras Universidades, en los montos que se dedican a esta actividad y en las constantes modificaciones institucionales que han afectado la investigación. Sin embargo, la función de investigación debe ser analizada desde una óptica académica para negociar la construcción de prioridades y de perspectivas para normar un proceso de avance coherente con las nuevas características del ambiente que envuelve a las Universidades y con las nuevas demandas sociales que se les plantean.

En la hora actual, la Innovación Tecnológica marca el ritmo de los tiempos modernos y las Universidades tienen frente a si un desafío ineludible. Ahora, la investigación no es más un proceso suntuario para el frívolo reconocimiento de prestigio de una institución universitaria. La investigación universitaria es una acción que coloca a las Universidades ante el dilema de ser simples extensiones de la acción escolar media-superior o de ser auténticos y legítimos actores implicados en la reconstrucción de la sociedad en la que les ha tocado vivir. No es exagerado decir que la respuesta que la Universidades ofrezcan a la Innovación Tecnológica definirá su papel y su relevancia como agentes del cambio social.

Es imprescindible que las Universidades reflexionen y analicen el carácter y sentido de sus acciones de investigación para que sobre esta base se reconstruyan internamente e influyan en sus entornos. Es obvio que una Universidad que internamente no se innova no es capaz de plantearse innovar la sociedad. Por esta razón fundamental, es necesario poner a discusión la capacidad de innovación tecnológica de la UAEM y buscar los elementos que contribuyan alimentar un debate que conduzca a negociar las vías por las cuales podría avanzar la investigación tecnológica en la UAEM.

Este trabajo pretende interesar a los lectores a la búsqueda de puntos teóricos de referencia de los cuales puedan discernirse metodología y técnicas para profundizar los estudios sobre las capacidades de innovación tecnológica en las universidades mexicanas.

Las respuestas a las preguntas mencionadas al inicio de este apartado demandan resolver problemas de orden teórico, metodológico y técnico. 
Entonces, una parte de nuestra problemática consiste en aproximarse a determinar la potencia de innovación tecnológica de la UAEM y, de otra parte, en explicamos que es la innovación tecnológica, cómo participa la Universidad en esta acción y, cómo se articulan la investigación, su marco jurídico y la acción de vinculación Universidad-Sector productivo.

\section{Antecedentes de la Investigación}

Existen una gran cantidad de estudios sobre la investigación en las Universidades, algunos evalúan la productividad científica en términos de sus indicadores (Viladiu et al. 1992), otros trazan la evolución de la investigación y las tensiones entre las funciones de enseñanza e investigación (Chazaro 1995), otros sobre las tendencias de la investigación (Victorino 1995), otros más sobre la vinculación Universidad-Empresa en diferentes experiencias (López y Solleiro 1992). También existen una gran cantidad de estudios sobre la capacidad de innovación de organismos, dependencias y programas de investigación (Arvanitis et al. 1986), sobre la evaluación de la investigación aplicada (Georghiou y Gibbons 1987); sin embargo, aún son escasos los trabajos abordan la capacidad de innovación tecnológica en las Universidades.

Los antecedentes formales de estudios de la capacidad tecnológica en las universidades mexicanas se iniciaron en los años 80. En este sentido, la primera experiencia en la representación de la capacidad tecnológica de una Universidad fue cuando el Centro para la Innovación Tecnológica (CIT) de la UNAM presentó el documento "Proyectos orientados a la producción de bienes y servicios” en 1985 (UNAM 1985). En realidad se trataba de una enumeración clasificada de los proyectos de investigación orientados a la producción de bienes y servicios que la UNAM consideraba socialmente útiles y disponibles para su transferencia.

De hecho, una parte de los estudios sobre la capacidad de innovación en México se han centrado en la evaluación de la actividad de vinculación del propio CIT (Lomnitz et al. 1988, Waissbluth et al. 1986, Esteva 1995). En cierta medida, en este trabajo se aborda la viabilidad de establecer en la UAEM una instancia de vinculación y transferencia de tecnología del tipo CIT.

\section{Fundamentación Teórica}

La fundamentación teórica se sustenta en la elaboración de conceptos ordenadores que dan cuenta de la Innovación en su origen, estructura y función; de la relación entre la concepción, desarrollo y utilización de los productos 
resultantes de la innovación, así como de los vínculos entre la Universidad y la Industria; principalmente.

Existen una gran cantidad de definiciones de Innovación, ${ }^{3}$ todas ellas coinciden en el carácter utilitario-mercantil que deben tener los productos de la investigación y desarrollo, En este trabajo se asume esta definición en tanto que permite distinguir la masa de resultados de Investigación y Desarrollo que no fueron exitosos en el mercado de aquellos resultados que fueron aceptados por los consumidores.

En otro sentido, las definiciones de Innovación recopila- das por Smith (1992) coinciden en separar la fase de investigación de la explotación comercial de los productos resultantes de investigación. Esta separación es parcialmente justa cuando se trata de abordar la capacidad de innovación desde la perspectiva de algún actor socio-técnico. En efecto, en la realidad no es posible romper las relaciones condicionales que favorecen o impiden el avance de la creación y el consumo y/o aplicación de innovaciones.

La popularidad de la distinción entre creación y aplicación de conocimientos radica en la fuerza cultural de Occidente que ha impuesto su racionalidad a casi todo el planeta. En efecto, esta distinción fue elaborada por los fundadores del pensamiento occidental como una separación entre la teoría y la técnica; particularmente, Aristóteles estableció la diferencia entre episteme

3 Keith Smith ha recopilado algunas definiciones dominantes sobre las categorías de innovación y de producto nuevo en algunos países europeos A continuación presentamos las más relevantes:"Germany By innovations are mant new features and marked improvements in productis and manufacturing and processing techniques, including information technology for office and administrative work. Product innovations are directed at new markets (reeds) and/or differ considerably in terms of technology form the products manufactured so faro Italy, Technological innovations means the conversion of and idea into a saleable, new or improved operational process used in the production of goods and services. It therefore involves a series of scientific, technical, financial and commercial activities. A technogically innovative product means a product giving a company access to a new market, whether completly new or new only to the firm, or a product showing substantial technological difference in the services, materials or components used compared with previous manufactured products. ...The innovation way concern an entire product if it is completely new, or a few parts or components modifying the features our uses of a complex product such as a telephone exchange, a machine tool or a vehicle. France, This enquiry is aimed at product innovations news products or significantly changed cid products. A product is considered as technologically innovative if it gives access lo a new market, or if it can be significantly distinguished from previously manufactured products in lerms of technology or the services wich it provides for a user. We are not concerned with innovations of a purely aesthetic or stylistic nature "(Smith, 1992: 384). 
haciendo alusión a la actitud teórica y contemplativa del mundo y la techne para distinguir la actitud humana que produce materialmente el mundo (Barone 1989). A pesar de la popularidad y fuerza social para separar creación y aplicación, en este trabajo no se comparte la división clásica de la innovación entre las fases de la creación y la aplicación de conocimientos y de objetos.

Las opiniones que inspiran este trabajo tienen tres fuentes. Primeramente, de Georges Simondon quien suponía que la cultura oculta en la realidad técnica la realidad humana y que frente a las técnicas, el Hombre tenía un deber análogo al que jugó por la abolición del esclavismo y por la afirmación del valor de la persona humana (Simondon 1989:9). En segundo lugar, las opiniones que animan este trabajo están cercanas a las de Marcel Mauss (Mauss 1927) quien rechazando las divisiones tajantes entre la cultura y la técnica y entre el conocimiento y la aplicación, fundo la escuela antropológica de la técnica. Según Mauss, la técnica es un «hecho social total», él integra en un sólo haz los utensilios, los usos y las representaciones. Así, hasta en el extremo analítico de la fundación cultural, la corporeidad del hombre se mezcla con las manifestaciones culturales dando por resultado distintas «técnicas del cuerpo» dependientes de cada cultura. Así por ejemplo, el caminar es diferente en cada cultura a pesar de cumplir con la misma función social de desplazarse de un punto a otro (Mauss 1927). Desde luego que la noción de <hecho social total $>$ no es compartida por muchos antropólogos posteriores a Mauss. De hecho, a excepción de André Haudricourt, casi todos los antropólogos han sucumbido a la tentación de separar la realidad en sus componentes ontológicamente purificados de Cultura y Sociedad y con ello aceptan la división entre conocimiento y aplicación técnica. Finalmente las numerosas "etnografías de laboratorios" de las nuevas escuelas de la sociología y la antropología de la ciencia y la técnica, han aportado elementos empíricos suficientes para refutar la separación definitiva entre la investigación básica e investigación aplicada o entre investigación e innovación (Callon 1981, Hughes 1983). A partir de estas fuentes consideramos que las únicas rupturas entre la investigación y la innovación son las divisiones ontológicas. En efecto, las tendencias disciplinarias de muchos tecnólogos y técnicos alimentan dos movimientos epistemológicos contrarios e irreconciliables: El primer movimiento consiste a explicar y a reducir la tecnicidad en las nociones ontológicas técnica y sociedad. Según esta noción, la técnica es la causa de la inmanencia de la tecnicidad humana de origen somático (Leroi-Gourhan 1945) o la trascendencia de la producción cultural (Perrin 1987, Basaglia 1991). Esta imagen de separación entre la técnica y la sociedad requiere un segundo movimiento que intenta simular la recuperación de las relaciones 
extraviadas. El segundo movimiento procura reconciliar técnica y sociedad por me- dio de supuestas relaciones recíprocas, de sentido contrario, dialécticas, etc. (Arellano 1996).Es posible evitarse los problemas de relacionar técnica y sociedad si no se recurre al expediente de separarles a priori, de allí que en este artículo se adopte una posición adisciplinaria de la innovación.

Adoptando una perspectiva adisciplinaria, se define la innovación tecnológica como un proceso continuo y sin rupturas que comienza con la fabricación de hechos científicos en los laboratorios científicos y concluye con el empotramiento y uso de Objetos Técnicos (OT) por los diferentes actores de la sociedad. Desde este punto de vista, la innovación tecnológica no tiene fronteras infranqueables entre las fases de Investigación, de Desarrollo y de Utilización ni entre la Fabricación y el Uso de nuevos artefactos.

En correspondencia con la perspectiva adisciplinaria que adoptamos, la categoría más importante en este estudio es el de Red socio-técnica (RST). Definimos una Red Socio-técnica como una estructura tecnológica relativamente estable, producida e integrada por realidades humanas y técnicas que distribuye las relaciones entre los actores sociales y natura- les. Esta categoría alude al carácter continuo e inseparable del proceso de innovación y a la imbricación que subyace a la estructura de los actores implicados en la innovación tecnológica.

Ahora bien, en la sociología y la antropología de la innovación existe una constelación de definiciones de RST. Así, la teoría de sistemas ha influenciado los estudios de la innovación y las definiciones de RST de Hughes (1983), en tanto que la teoría Relativista-constructivista ha inspirado a Pinch (1985), Bijker y otros (Bijker et al. 1993) sobre el papel crítico de los grupos sociales en la definición y solución de problemas que impiden el desarrollo de un artefacto.

Gracias a estos estudios se han precisado los elementos que participan en la innovación y el carácter social de la innovación. La noción de grupos técnicos de interés que ha desarrollado Bijker, ha puesto en evidencia; a) los diversos intereses científicos, técnicos y económicos que sostienen los actores en la determinación de una trayectoria tecnológica, b) la integración de un amplio y complejo grupo de actores sociales que interactúan para tratar de imponer sus perspectivas y opiniones al conjunto de actores de una Red y, c) que una innovación es estabilizada (Bijker et al. 1993) o que es puesta en «caja negra» (Pinch 1985).

Por su parte Hughes tiene otra versión de RST según la cual en la construcción de sistemas tecnológicos los constructores no respetan ninguna 
frontera disciplinaria o cognitiva; además que los compuestos de un sistema o los actores están ligados y determinan la adición de objetos, de innovaciones, principios científicos, especialistas y de fuentes financieras en un poderoso sistema holístico (Hughes 1983).

El acercamiento de Pinch y Bijker no aclara la fusión de intereses sociales con las leyes naturales que rigen el funcionamiento de los artefactos y el acercamiento de Hughes no precisa la estructura y jerarquía de los elementos de orden distinto que se agregan (adicionan, en palabras de Hughes) en un sistema tecnológico. En este trabajo se toma como punto de referencia la Teoría del actor-red que ha sido expresada por Law (Law 1984) y que ha tomado una forma más detallada en Callon (1986) y Latour (1986).

La hipótesis fuerte de la teoría del actor-red abandona la distinción entre actores humanos y fenómenos naturales. Según esta idea, los artefactos son la mezcla de esas entidades diferentes que toma forma de red y que actúan como verdaderos actores. Refiriéndose a las técnicas, Law indica que «luego que un actor (artefacto o innovación) define y construye su mundo, él define y pone en relación toda una serie de cajas negras llámense seres humanos, partículas elementales o firmas petroleras, en todo caso, es legítimo llamar actor-red a tal conjunto» (Law 1984:121). Según Callon, los artefactos tienen una naturaleza paradójica, ellos son construidos por la coproducción de aspectos sociales y naturales pero que en el momento final de su construcción son separados por los humanos en aspectos sociales o naturales (Callon 1986).

Una RST es un compuesto de realidades sociales y naturales que se encuentran mezclados de manera inseparable, esta naturaleza otorga a los OT (artefactos) una estructura híbrida que se extiende simétricamente tanto en la Naturaleza como en la Sociedad (Arellano Hernández 1996). Una RST mantiene en copresencia la sociedad, los objetos y los textos. De esta forma se considera que una innovación tecnológica construye relaciones inéditas y únicas entre las cosas y los hombres.

La breve discusión anterior sobre la RST nos permite enunciar una definición de capacidad de innovación, como la noción que alude a la potencia de un actor, o de una RST para rendir con eficiencia y eficacia resultados tangibles en el proceso de generación, desarrollo y aplicación social de nuevos conocimientos y procesos tecnológicos. Así innovación tecnológica exige la presencia y sincronía de dos procesos complementarios. De un lado, la maduración de los ámbitos científico-técnicos, empresarial y administrativo; por el otro, la articulación de esos ámbitos en una cadena operatoria (« chaine operatoire » en palabras de Mauss) en sentido de sistema socio-técnico estabilizado (Red Socio-técnica). 
La innovación tecnológica es un proceso social que involucra todos los sectores e instituciones de los países. A la hora actual, ningún actor social está al margen de los procesos y fenómenos científico-técnicos. En este sentido la innovación tecnológica es un proceso complejo que tiene-como actor ineludible a la Universidad.

La capacidad de innovación tecnológica de una Universidad seria la posibilidad que esta tiene para ofrecer a los sectores productivos Objetos técnicos susceptibles de for- mar e integrarse como RST.

La capacidad de innovación se manifiesta en las dimensiones simbólica, material y social. La dimensión simbólica se refiere a los elementos narrativos que sincronizan y regulan las gramáticas culturales de los actores. En este sentido, el marco jurídico y las acciones legítimas encuadran la capacidad de innovación tecnológica.

La dimensión material se refiere a la infraestructura con la cual es posible realizar actos y gestos tecnológicos para acrecentar la eficiencia de los procesos técnicos o para desarrollar nuevos productos. Esta infraestructura tiene una parte humana y otra estrictamente material. El savoir-faire (Know-how) del personal que participa en la innovación es un supuesto material para cualquier esfuerzo de generación desarrollo y difusión de nuevos conocimientos científico- tecnológicos, de igual forma, la infraestructura en laboratorios, equipos de investigación y recursos financieros son una referencia para el desarrollo de la innovación.

\section{La dimensión social se refiere a la organización y jerarquización de los actores en el proceso de la innovación}

Para el caso presente, se distinguen algunos elementos estratégicos de las dimensiones simbólica, material y social en el actor Universidad a fin de estudiar la capacidad para la innovación tecnológica. Para ello se toma como caso de estudio a la Universidad Autónoma del Estado de México y se abordan las dimensiones en que se manifiesta la innovaciones en el marco jurídico-legal de la UAEM como componente decisivo de la dimensión simbólica; a la información de los proyectos de investigación aprobados por la Coordinación General de Investigación Científica y de los investigadores responsables como componentes fundamentales de la dimensión material y a organización de la investigación en Centros, Escuelas y Facultades así como la operación de la Oficina de vinculación de la UAEM como componentes estratégicos de la dimensión social de la innovación. 
Nos interesa conocer la participación de la UAEM, vista como actor de innovación tecnológica. La caracterización de las áreas de investigación tecnológica, los niveles de desarrollo de los proyectos de investigación, el estudio de la legislación universitaria vigente y el funcionamiento de la oficina de vinculación universitaria son los elementos empíricos que permitirán interpretar la capacidad de innovación de la UAEM.

Con este trabajo se pretende identificar las fuerzas que posee la UAEM para convertirse en un actor de la innovación regional. En este sentido, reconocemos dos limitaciones en el trabajo; a) al interior de la institución no se enfatizan las debilidades o los aspectos que presentan obstáculos a la capacidad de ofrecer productos y/o servicios de calidad y b) al exterior de la institución no se estudian las oportunidades ni las amenazas a la capacidad de innovación tecnológica (ver nociones de debilidades, obstáculos, oportunidades y amenazas en: García-Torres 1990).

\section{Estrategia Metodológica}

La estrategia metodológica que se adopta en el estudio corresponde al punto de vista de un evaluador. En efecto, luego que se fija una noción teórica de innovación se toma como un objetivo a lograr ("deber ser") por los actores implicados y ese giro metodológico es el que permite utilizar una serie de indicadores y de técnicas de investigación para conocer el grado en que los resultados de la actividad de investigación de la UAEM corresponde con el objetivo de la Innovación tecnológica.

La evaluación tradicional del proceso de innovación consiste en el estudio, la calificación y la medida en que los objetivos y metas enunciados de un programa se cumplen y las razones que se pueden argüir para explicar el grado de su cumplimiento o incumplimiento.

Este ejercicio se conforma de tres tareas. En la primera se fijan las nociones que corresponden con el "deber ser" de la innovación tecnológica de una institución universitaria mexicana (ver Fundamentación teórica). En la segunda se procura calificar y medir las acciones de investigación en la UAEM y en la tercera se hace una reflexión sobre el grado de cercanía que existe entre el "deber ser" de la innovación tecnológica con las acciones que se realizan en la UAEM.

En el fondo, el punto de vista evaluador que se adopta en el trabajo no se distingue grandemente del trabajo de investigación científica tradicional. En ambas se llevan a cabo dos pasos. En un primer paso, se fijan las nociones teóricas que orientan el trabajo de investigación, en un segundo paso se realiza la 
búsqueda y la elaboración de los datos que dan o no cuenta de las nociones y categorías que orientaron el trabajo de investigación.

Las técnicas utilizadas en este trabajo corresponden a cada una de las dimensiones de estudio (social, simbólica y material). En el caso del estudio del marco jurídico se utiliza la técnica del análisis de contenido. En el caso de la actividad de investigación se utilizan las técnicas de la investigación de estadística social y el programa FoxPro para explotar una base de datos sobre las características de los proyectos de investigación proporcionadas por la CGIEA de la UAEM. En el caso de la función de vinculación se realizan entrevistas, mismas que se aplicaron a los funcionarios responsables de esta actividad.

\section{La Capacidad de Innovación Tecnológica de la UAEM}

Antes de abordar la Capacidad de innovación tecnológica en la UAEM es importante dar algunos antecedentes y trazar una brevísima evolución de la función de investigación en esta institución. Posteriormente se abordaran tres aspectos de la innovación tecnológica: El conocimiento del contexto jurídico, la investigación en la UAEM y la cooperación UAEM-Sector productivo.

\section{Antecedentes de la Investigación en la UAEM}

Dentro del proceso de formalización de las actividades académicas de la UAEM se abre un espacio para la investigación en 1960, cuatro años después de transformarse de Instituto a Universidad, con la creación del Instituto de Investigaciones Sociales (CIC 1983).

En este primer intento la investigación tecnológica no fue contemplada, principalmente porque en ese momento los intereses de la administración universitaria se concentraban en la búsqueda de fórmulas de acción para resolver la problemática social del Estado de México. Por lo tanto las áreas que se contemplaron dentro del Instituto fueron: sociología, medicina social, economía y trabajo, ingeniería y arquitectura social e historia (CIC 1983).

Posteriormente se consideraron otras áreas de investigación diferentes a las ciencias sociales, específicamente con la creación del Comité de Investigación Científica de la Escuela de Medicina en 1969 (CIC 1983).

Otro organismo que nace en el marco de la investigación es el Centro de Investigación, Cálculo e Informática (CICALI) en 1975 (CIC 1983), su propósito era dotar a los investigadores universitarios de herramientas tecnológicas. Pero la investigación de los procesos tecnológicos no fue conside- 
rada dentro de los objetivos de este Centro, prueba de ello son los primeros trabajos relevantes del CICALI enfocados al establecimiento de sistemas informáticos de selección de aspirantes, de controles escolares, de nóminas de pagos, etc.

El esfuerzo formal e institucionalizado para organizar los procesos de investigación en la UAEM se presenta en 1977 con la creación de la Coordinación de Investigación Científica (CIC) (CIC 1983). Esta coordinación promovía la investigación en facultades, escuelas y dependencias de la UAEM. Pero es hasta 1981 cuando sus actividades se con- solidan con la formulación de un Plan de Trabajo, en donde se contempla una serie de programas que, si bien no adquieren un carácter científico y menos aún tecnológico, sientan las bases para establecer la organización y control de los procesos de investigación.

En el transcurso de la organización administrativa de la investigación, la administración para el periodo 1985-1989, se lleva acabo la formación de los Centros de Investigación por área de conocimiento. Se trataba de integrar a los investigadores adscritos a la UAEM que realizaban hasta ese momento su trabajo en forma asilada. Se deseaba, además, imprimir mayor dinamismo a las áreas que no habían sido consideradas en el ramo de la investigación universitaria. Dentro de estas áreas se encontraba la investigación tecnológica, lo que dio nacimiento al Centro de Investigación en Ciencias de Arquitectura, Ingeniería y Tecnología. Los Centros de Investigación formuladas en el Plan de Trabajo 1985-1989 fueron: 1. Centro de Investigaciones Cerebrales CICE, 2. Centro de Investigación en Desarrollo Odontológico CIDO, ${ }^{4}$. Centro de Investigación en Ciencias Agropecuarias CICA, 4. Centro de Investigación en Ciencias de Arquitectura, Ingeniería y Tecnología CIAIT, 5. Centro de Investigación en Ciencias Económicas Administrativas CICEA, 6. Centro de Investigación en Ciencias Sociales y Humanidades, 7. Centro de Investigación en Ciencias de la Salud CICS, 8. Centro de Investigación en Ciencias Básicas CICB.

Con base en el trabajo de los Centros de Investigación, la administración universitaria en el período 1989-1993 instrumenta dentro de su plan de trabajo una delimitación más precisa por área de investigación. Este plan se sincroniza en la política nacional de investigación que planteaba la organización por Academias de especialistas. La UAEM pro- mueve la organización de la investigación universitaria a través de academias (CGIC 1990); las academias se formaron en ocho campos de investigación: 1. Ciencias ambientales,

4 Cabe mencionar la existencia del Estatuto Universitario de la UAEM el cual fue aprobado en Junio de 1996, su difusión no está totalmente formalizada, por esta razón no abordamos su contenido. 
2. Ciencias de la salud, 3. Educación superior, 4. Estudios de la población, 5. Historia, 6. Asentamientos Humanos, 7. Flora y fauna, 8. Regional de vivienda. Es evidente que en la investigación tecnológica esta ausente de este esquema académico. La vida de estas academias es efímera y se extingue con el abandono del Gobierno Federal de esta forma de impulsar la investigación.

En esta evolución de la investigación es necesario resaltar la creación del Fondo para la Investigación Científica y Tecnológica (FONDICT), que actualmente financia 221 proyectos de investigación con fondos propios (Morales 1995).

En resumen puede decirse que dentro de todos los procesos de institucionalización de la investigación, la UAEM no ha tenido un espacio institucional formal para impulsar la investigación en tecnología.

\section{Legislación Universitaria e Innovación}

En esta apartado se estudia la legislación universitaria vigente desde una perspectiva que la indaga su relación con la innovación tecnológica.

La legislación de la UAEM tiene seis instrumentos fundamentales. La Ley de la UAEM, El Reglamento General de la UAEM, El Reglamento de Facultades y Escuelas profesionales, El Reglamento de Personal Académico, El reglamento de Escuelas Preparatorias y El Reglamento de integración y funcionamiento del Consejo Universitario de la UAEM.

a) La Ley de la UAEM $^{5}$ asegura en su objeto la generación, la transmisión y la extensión del conocimiento universal. Dentro de sus fines se asegura la investigación humanística, científica y tecnológica (Artículo 20). Estas dos definiciones fundamentales corresponden a la investigación y a la extensión: por investigación se entiende «el ejercicio creativo de los integrantes de la comunidad que genere, rescate, preserve, reproduzca y perfeccione el conocimiento universal. En el marco de la libertad de investigación se vinculará a los problemas estatales, regionales y nacionales» (Artículo 15o). Por difusión cultural y extensión universitaria se entiende la actividad que relaciona a la Universidad con la sociedad y «pone a su disposición de ésta el resultado de su trabajo académico» (Artículo 160). En el marco de las definiciones generales que corresponden con el de la Ley de la UAEM no se aprecia ninguna limitación a la innovación, es una ley sobria y no excluyente de ninguna de las funciones sustantivas de la Universidad.

5 Promulgada el 3 de marzo de 1992 
b) El Reglamento General de la $\mathrm{UAEM}^{6}$, establece que la autoridad máxima de las Escuelas y Facultades es el Consejo de Gobierno (CAPITULO V, DE LOS CONSEJOS DE GOBIERNO). En este tenor, el Consejo Académico (CAPITULO VI, DE LOS CONSEJOS ACADÉMICOS) es un órgano de consulta Este consejo académico «tiene entre sus facultades 1 . Estudiar y dictaminar los proyectos e iniciativas de carácter académico que les presente el Rector, el Director, los miembros del Personal Académico y los alumnos, o los que surjan en su seno (Fracción 1, Artículo 41o). 2. Dictaminar y participar en los programas de coordinación general de investigación, a través de la dependencia respectiva de la Universidad» (Fracción 111, Artículo 41o).

De lo anterior puede concluirse que la organización de la investigación en las Escuelas y Facultades no está adecuadamente organizada y que la función de extensión y vinculación con la sociedad esta simplemente ausente. Pareciera que una dependencia de la Universidad, digamos la Coordinación de Investigación Científica, coordina la investigación y que las Escuelas y Facultades participan en los programas que de aquella se derivan (No se específica porqué de esta falta de organización, ni el papel de la CIC).

En el mismo reglamento y particularmente en el TÍTULO CUARTO, DE LA INVESTIGACIÓN y en el TÍTULO QUINTO, DE LA DIFUSIÓN CULTURAL y EXTENSIÓN UNIVERSITARIA se explica escuetamente que: la investigación científica en la Universidad «se llevará a cabo en las dependencias académicas mencionadas en el artículo 440 de este Reglamento (Es un listado de 16 Escuelas y Facultades que incluyen a la Preparatoria), de acuerdo con planes específicos. La investigación contará con los institutos y centros de apoyo necesarios» (Artículo 51o). Asimismo que la Difusión cultural y la extensión universitaria consistirán en: «l. Difundir la cultura en el ámbito de la Universidad y de la sociedad y, 11 Estructurar y formar programas de difusión cultural y extensión que lleven beneficios a la comunidad universitaria y a la sociedad $\gg$. Esta descripción sucinta de las funciones de investigación, de difusión cultural y de extensión contrasta con la descripción minuciosamente detallada de la Planeación en la Universidad, descrita en 5 artículos (Artículos 51o a 55o).

6 Del 23 de marzo de 1980. 
c) Reglamento de Facultades y Escuelas Profesionales de la UAEM ${ }^{7}$ dentro de este documento es remarcable que el Consejo de Gobierno (máxima autoridad al interior de Escuelas y Facultades) nombrará comisiones permanentes o especiales para el estudio de los asuntos de su competencia y que este consejo tendrá por lo menos dos comisiones permanentes: «La Comisión de Estudios de Licenciatura y la de Estudios de Posgrado» (Artículo 160). En las comisiones permanentes del Consejo Académico se reitera que este órgano también tendrá las Comisiones de Estudios de Licenciatura y de Posgrado. En este Reglamento no se contempla la creación de comisiones sobre la investigación y/o la vinculación.

Paradójicamente a la normatividad de las Comisiones permanentes de los consejos de gobierno y académico, el Reglamento instituye dos coordinaciones: La de investigación y la de Difusión cultural y extensión universitaria (CAPÍTULOS VI y VII). La creación de estas coordinaciones marcará un hito en el desarrollo de la investigación pues estas posiciones serán la base para la conformación de un Consejo universitario de investigación en el año de 1989. Ahora bien, el Reglamento de Facultades y Escuelas Profesionales de la U AEM aborda la manera de ejercer la investigación, lo cual es plausible. Sin embargo, declara reiteradamente que la investigación es de carácter científico (TITULO QUINTO, DE LA INVESTIGACIÓN CIENTIFICA), estando ausente la investigación tecnológica. En el Artículo 211 o esta ausencia de la investigación tecnológica se potencia pues en ninguna de las 4 fracciones se declara otra forma de investigación que no sea la científica ${ }^{8}$ y sólo en la última fracción se indica que la investigación científica debe participar en la solución de problemas tecnológicos, entre otros.

Este Reglamento contempla con suficiente rigor científico los aspectos que deberán contener los proyectos de investigación. Este esfuerzo es loable pero el protocolo que se reglamenta es típico para un proyecto de investigación científica. ${ }^{9}$ Para que no existan dudas de estas afirmaciones

7 Aprobado el 8 de mayo de 1984.

8 A continuación transcribimos los artículos 210o Y 211 o para ilustrar el sesgo científico de la investigación: ARTICULO 210o. La investigación científica que se lleve a cabo en las Facultades y Escuelas Profesionales, se regirá por las normas contenidas en este título y demás disposiciones aplicables. ARTICULO 211o. La investigación científica tendrá por objeto: I Generar nuevos conocimientos científicos o transformar los existentes. II. Apoyar los objetivos de los planes y programas de estudio. III Estimular la formación, actualización y perfeccionamiento del personal de investigación. IV. Participar en las resolución de los problemas científicos, tecnológicos y soci

9 Por ejemplo el punto 4 del protocolo señala que se deberá describir «el tema o la prob- 
científicas, en el artículo 219 o se declara que al concluir el proyecto de investigación, el investigador deberá presentar por escrito un informe final que comprenderá un reporte técnico financiero y un ensayo sobre la investigación (Artículo 219o). Para culminar con el tema de la investigación científica, el Reglamento de Facultades y Escuelas Profesionales de la UAEM aborda, en el artículo 220o, sobre los derechos de autor pero en ningún artículo se abordan los derechos sobre las patentes o sus licenciamientos generados por los investigadores.

En relación a la función de Difusión cultural y Extensión universitaria, y por extrapolación de lo reglamentado en la función de investigación, en este Reglamento se declara por objeto de la Difusión «la transmisión entre la Comunidad universitaria y la Sociedad, los conocimientos de carácter científico, técnico y humanístico (el subrayado es nuestro) ». Además, se precisan las actividades culturales (Artículo 2260) pero no las de difusión o vinculación relacionados con la tecnología.

d) En el Reglamento de Escuelas Preparatorias se establece la función de investigación declarando los siguientes propósitos: ARTÍCULO 4o. La Escuela Preparatoria coadyuvará a la realización de los fines de la Universidad mediante las siguientes actividades: I. Planear, organizar, dirigir, impartir, vigilar y evaluar los estudios de bachillerato. II. Promover e impulsar la investigación, de acuerdo a las características del nivel bachillerato. III. Promover e impulsar la difusión cultural y extensión universitaria, de acuerdo a las características del nivel bachillerato. Este reglamento es demasiado laxo y desde luego no explícita la promoción y el impulso a la investigación tecnológica.

e) Reglamento de Personal Académico de la UAEM, se inscribe que una de las funciones del personal académico es la de realizar investigación científica (Artículo 4o, fracción II). Este Reglamento es importante, toda vez que en él se abordan los aspectos de regalías por conceptos de derechos de autor y de propiedad industrial. En el artículo 5o se declara «el derecho del Personal académico a su participación por concepto de derechos de autor o de propiedad industrial, según la legislación federal vigente (Artículo 50, fracción $\mathrm{X} \gg>$. Esta importante fracción es ambigua pues no aclara el porcentaje de las regalías respectivas que recibirían los miembros del Personal académico. El Artículo tampoco aclara a cual legislación federal vigente habría que remitirse para interpretar la recepción de regalías.

lemática a tratar». (Articulo 215o) 
En la legislación federal vigente se encontró que en la Ley Federal del Trabajo y de manera precisa en el artículo 163 se aclaran los derechos de los inventores en el caso de los trabajadores; sin embargo la inexistencia de patentes y de licencias en la UAEM no ha planteado la necesidad de interpretar de alguna manera esta legislación.

En otro orden de ideas, el Reglamento de Personal Académico de la UAEM considera el procedimiento de Juicios de promoción para dictaminar las promociones salariales y categorías del Personal académico. En estos juicios se toma en cuenta la calificación de los factores de eficiencia, preparación y antigüedad laboral en la UAEM (Artículo 53o).

En el factor de eficiencia se evalúa la responsabilidad, la colaboración a la institución, la puntualidad y la asistencia al trabajo. El factor de preparación evalúa el nivel de estudios, la producción académica, la experiencia y los cargos de coordinación o dirección académicas desempeñados por el Personal académico. En realidad, en el aspecto de la producción académica se puede leer el sesgo científico de esta producción. ${ }^{10}$ De esta manera, ninguno de los 12 elementos considerados evoca la producción tecnológica; el único elemento posible de evaluación de la producción tecnológica se refiere a «Otros proyectos académicos» que tiene un rango de valor entre 1-10 puntos y que puede equipararse con el elemento «Elaboración de programas de estudio»u «Otras traducciones» que representan apenas el $50 \%$ del valor de un artículo especializado (entre 5-20 puntos).

f) Reglamento de Integración y Funcionamiento del Consejo Universitario de la UAEM, aquí se contempla la existencia de 8 Comisiones Permanentes (CAPITULO CUARTO, DE LAS COMISIONES) dentro de las cuales la fracción I corresponde a la Planeación y Evaluación Académica. Esta comisión tiene como facultades y obligaciones la de «Analizar y dictaminar sobre el Plan General de Desarrollo de la Universidad y todos los proyectos vinculados con la planeación y evaluación de la docencia, investigación y difusión de la cultura» (Artículo 430). Aquí se olvida la función de la Extensión universitaria.

De la lectura de los 6 instrumentos de la legislación vigente se puede con-

10 En la fracción 11 del articulo 59o sobre la calificación de la producción académica se consigna que el puntaje por productos de investigación es: Articulo especializado 5-20, Capítulo para libro 5-20, Antología 10-25, Libro 20-80 Traducción de libro 10-40, Otras traducciones 1-10, Elaboración de notas o materiales didácticos 5-20, Conferencia 1-4, Investigación concluida 20-100, Coordinación u organizo de eventos académicos 1-7, Elaboración de programas de estudio 3-10. Otros proyectos académicos 1-10, 
cluir que ella es un marco legal orientado a la promoción y desarrollo de la investigación científica pero que no es la adecuada para encauzar la innovación tecnológica. Asimismo que respecto a la Vinculación y la transferencia de tecnología no existe claridad legal y, finalmente, que la legislación de la Difusión y la Extensión se sesga hacia la promoción de las manifestaciones culturales no tecnológicas.

Ante este vacío de legislación en favor de la innovación tecnológica surge la pregunta: ¿por qué la legislación de la UAEM hipostasia la investigación científica en detrimento de la innovación? Según este punto de vista, se trata de la correspondiente falta de los elementos materiales y sociales que no permiten estructurar una Red de innovación tecnológica. En efecto, la lectura sociológica de la ausencia de una legislación en pro de la innovación indica exactamente la falta de actores científicos y de estructuras institucionales asociados a la innovación. Dicho de otra manera, no hay los suficientes y potentes investigadores de tecnología quienes creando situaciones de patentamiento o de licenciamiento impriman su sello en la legislación. Tampoco hay los gestores de tecnología quienes incrustados en la administración de la Universidad influyan en la reorganización de la investigación y de la legislación.

\section{La Investigación en la UAEM}

Actualmente la UAEM destina a la investigación $11.1 \%$ de su presupuesto, lo que representa 37.9 millones de pesos (Morales 1995). Esta cifra es muy superior a los años anteriores, sin embargo todavía representa la mitad del gasto para apoyo académico-administrativo (22.8\% del gasto universitario).

La UAEM tiene en su sistema dependiente 31282 alumnos inscritos, de los cuales 1553 pertenecen al nivel de maestría, esto equivale a $4.9 \%$. Respecto a los programas de doctorado, no existe el dato exacto de los alumnos inscritos ya que los tres programas que ofrece la UAEM son de reciente creación. Si se toma en cuenta que existen registrados 271 proyectos de investigación resulta que la población de estudiantes en posgrado no están vinculados a estos proyectos (habría $\mathrm{O} .17$ proyectos por estudiante o 5.7 estudiantes por proyecto). Indudablemente, el fenómeno anterior refleja la desvinculación entre investigación y posgrado en la UAEM y el desperdicio de potencial de investigación por parte de los estudiantes de posgrado.

En relación al personal académico, en la UAEM existen 3091 personas. De este total 783 tienen grado académico (Morales 1995). Según el número de proyectos de investigación existen 237 miembros del personal 
académico responsables de proyecto, esto significa que el personal que realiza investigación en la UAEM representa $7.6 \%$ de todo el personal académico y $30 \%$ del que cuenta con algún grado académico. Se desconoce el perfil del personal que no realiza investigación, sin embargo puede indicarse que $70 \%$ del personal con grado académico que no realiza investigación representa un potencial que no es explotado por la institución.

La investigación se realiza en 32 organismos integrados ${ }^{11}$ (Morales 1995: inv-01). Existen 59 proyectos con financiamiento del CONACYT y del Gobierno Federal y 221 con financiamiento de fuentes propias (Anexo 1). Esto habla de la voluntad política de la UAEM por verter una parte importante de recursos provenientes de sus ingresos propios en la Investigación. A diferencia de otras universidades públicas, la UAEM tiene recursos para realizar investigación por si misma, pero no tiene una política clara que le permita dar dirección a esta función. Esto es importante puesto que la UAEM tiene un margen de maniobra menos restringido que el de otras universidades para guiar la investigación de conformidad con su política interna. La disponibilidad financiera para apoyar la investigación de la UAEM es una potencialidad que se puede encauzar hacia la innovación tecnológica.

Si se toma como índice el número de proyectos autorizados por la Coordinación General de Investigación y Estudios Avanzados (CGIEA) en los últimos 7 años se observa que la evolución de la investigación científica y tecnológica en la UAEM muestra un crecimiento espectacular pasando de 4 en 1989 a 119 proyectos autorizados en 1995 (Cuadro 1).

El área de ingeniería y tecnología (columna 3) tiene un crecimiento igualmente importante, sin embargo el número de proyectos representa solamente $3.32 \%$ del total de proyectos autorizados en 1995 (ver columna t). $12.5 \%$ del total de proyectos corresponden según los criterios de CONACYT a proyectos de ingeniería y tecnología, en tanto que en relación al tipo de proyectos $9.9 \%$ corresponden a los de tecnología.

En general la investigación en la UAEM se encuentra dispersa. Tomando en cuenta el número de Organismos académicos que realizan investigación se tiene que en promedio cada uno de ellos realiza 8 investigaciones. Desde luego que la consolidación de la investigación varía en cada organismo; por ejemplo, en $50 \%$ de las dependencias universitarias el número de proyectos no rebasa los 4 proyectos, mientras que en 13 de ellas el número de proyectos supera el número de 8 (figura 1). En realidad puede decirse que la investigación tiene una cierta coherencia organizativa y numérica de sus proyectos en los Centros de investigación y particularmente en los de Ciencias Agro11 De acuerdo a la reglamentación de la UAEM se denomina "organismos y dependencias" a escuelas, facultades y centros de investigación de la Universidad respectivamente. 
pecuarias (CICA, 15 proyectos sobre agricultura campesina), Salud animal (CIESA, 13 proyectos sobre salud animal), Interamericano de recursos del agua (CIRA, 9 proyectos sobre recursos hídricos) y el de Ciencias Sociales y humanidades (CICSH, 9 proyectos sobre filosofía, política e historia). En las escuelas y facultades sobresale la investigación de la Facultad de Medicina (37 proyectos), de la Facultad de Humanidades (24 proyectos), de la Facultad de Química (21 proyectos), de la Escuela de Ciencias (18 proyectos), de la Facultad de Ciencias Agrícolas (14 proyectos), Ciencias de la Conducta (11 proyectos) y de la Facultad de Ciencias Políticas, Arquitectura y Arte (con 8 proyectos cada una) (Ver anexo 2)

De conformidad con las áreas del conocimiento que han sido diseñadas por el CONACYT, Los organismos que realizan investigación tecnológica son solamente 7: El Centro de investigación en C. Agropecuarias, el Centro Interamericano de Recursos del Agua, la Escuela de Ciencias, la Facultad de Arquitectura y Arte, la Facultad de ingeniería, la Facultad de Planeación y la Facultad de Química (Anexo 3 y Figura 2).

La posibilidad más importante en cuanto a la capacidad de innovación se localiza en las ingenierías, la química, la investigación hídrica y la agropecuaria. En las otras tres dependencias la investigación se encuentra dispersa.

Las áreas que destacan en las principales dependencias son: a) en la Facultad de ingeniería la metalurgia, la robótica, la construcción (ingeniería civil) y la energía calorífica. b) en la Facultad de Química destacan la obtención de substancias farmacéuticas, el manejo ecológico de substancias y la química industrial. c) En el Centro Interamericano de Recursos del Agua se concentran investigaciones de tratamiento de aguas residuales, de contaminantes en el agua y estudios sobre eventos hidrológicos extremos (Ver anexos 4 y 5 ).

En ciertos organismos de la UAEM se percibe un esfuerzo de sistematización y de organización de la investigación tecnología en líneas y programas definidos. Así la investigación que realiza el Centro de investigación en Ciencias Agropecuarias que aglutina su investigación en el desarrollo de innovaciones para la agricultura campesina, En el Centro de investigación y estudios avanzados en Salud animal sobre detección de elementos y compuestos contaminantes así como de enfermedades de las especies más comunes en la región de los Valles Altos del Estado de México. En la Escuela de Ciencias se encuentra un conjunto de investigaciones sobre elementos contaminantes y de estudios de la flora. En el caso de la Facultad de Ciencias Agrícolas encuentra un grupo de investigadores que desde la disciplina del fitomejoramiento ensayan diferentes variedades de trigo, maíz y papa para su difusión en la región de influencia de esta Facultad. 
De la lectura de la investigación en la UAEM puede apreciarse que su potencial de innovación tecnológica se encuentra en las Facultades de Ingeniería, en el Centro Interamericano de Recursos del Agua, en la Facultad de Química, en el Centro de Investigación en Ciencias Agropecuarias y en la Escuela de Ciencias. Es aquí donde se concentra la mayor experiencia de investigación en innovación tecnológica y donde los grupos de investigación constituyen un recurso potencial de la UAEM.

Según la Dirección General de Extensión y Vinculación, hasta ahora en la UAEM no se han hecho transferencias de tecnología, ni se ha solicitado el registro de patentes. En una investigación posterior hará falta adentrarse entre los investigadores para conocer en detalle las relaciones que realmente mantienen con el sector productivo.

Hay los elementos necesarios para afirmar que la función de investigación no conforma una red de investigadores universitarios, de temas de investigación, de estudiantes de posgrado con la investigación, y de un público interesado en los productos que pudiesen resultar de las investigaciones. El $70 \%$ del personal académico con posgrado no se integra a la red de investigación universitaria y no se conforma una política de investigación que permita tejer los hilos de una autentica Red de investigación. Por tal motivo no es exagerado decir que la UAEM es un actor al que le falta madurez para incorporarse al ejercicio de la innovación tecnológica.

\section{La Vinculación Universidad-Sector Productivo en la UAEM}

La vinculación universitaria se lleva a cabo en dos dependencias diferentes. De un lado existe el Programa de Vinculación dependiente de la Coordinación de Investigación y Estudios Avanzados y, de otro lado, la Dirección General de Vinculación y Extensión Universitaria que de- pende directamente de la Rectoría.

\section{Programa de Vinculación Universidad-Empresa de la Coordinación General de Investigación y Estudios Avanzados.}

El Programa de vinculación (PV) ubicado en la CGIEA de la UAEM tiene sus orígenes en la Dirección de investigación tecnológica de la misma CGIEA en la administración rectoral de 1989-1993 de la UAEM (Hernández 1996:c.p). En el rectorado actual se crea el Programa de vinculación dependiente directamente del Coordinador de investigación y estudios de posgrado. 
A comienzos de los años 90, la vinculación en la UAEM trató de seguir la corriente de creación de incubadoras (Empresas de Base Tecnológica, EBT) en las universidades mexicanas; sin embargo en la UAEM esta corriente de vinculación no cristalizó (Hernández 1996:c.p.).

Dada la competencia entre las dependencias de la UAEM por ejercer la vinculación universitaria, el Programa de vinculación enmarca sus acciones de vinculación específica a la investigación tecnológica derivada de los proyectos de investigación (Hernández 1996:c.p.). El PV conduce tres acciones principales. 1. El seguimiento del Programa ALFA (América Latina Formación Académica) de la Comisión Europea en la cual el Centro Interamericano de Recursos del Agua (CIRA), que depende de la Facultad de Ingeniería, encabeza la Red HYDROCIYTEC. 2. La animación del Proyecto del Programa del Medio Ambiente para la creación del Instituto Estatal del Medio Ambiente y, 3. La promoción en la UAEM del Programa de Enlace Academia-Empresa que depende de la Dirección adjunta del CONACYT.

En este último sentido, se tienen dos proyectos planteados. El del financiamiento del Centro de Diagnostico Genético que sería un centro de apoyo a las instituciones de Salud del Estado de México para los servicios de Genética Clínica, de Diagnostico Citogenético y Prenatal así como el de Laboratorio de Diagnóstico Molecular. De la misma manera se tendría el proyecto sobre la Planeación, diseño y operación de los sistemas de transporte que sería un programa conjunto entre el Instituto Mexicano del Transporte y la Facultad de Ingeniería.

Adicionalmente, el Programa de vinculación tiene a su cargo la vinculación de los proyectos alimentarios y educativos del Fondo de Ciencia y Tecnología de la UAEM (FONDICT). También se da seguimiento a una innovación de proceso para el cultivo de una seta que ha desarrollado la Escuela de Ciencias y que un empresario está interesado en adquirir (Hernández 1996: c.p.).

El Responsable del Programa de vinculación informa que la CANACINTRA, La empresa ASPROS, la Asociación de Productores de Leche y algunos empresarios aislados han tomado contacto para buscar investigaciones dignas de ser desarrolladas en sus plantas industriales. La CANACINTRA se ha interesado en la adquisición de Esquemas de administración desarrollados en la Facultad de Administración. También se han dado asesorías para la reorientación de dos proyectos de la Facultad de Ingeniería y uno de la Facultad de Química. A pesar de los esfuerzos que se hacen, este programa es orgánicamente débil, de un lado funciona con una sola persona (el responsable) y de otra la ubicación del puesto no tiene mayor relevancia en la CGIEA. 


\section{Dirección General de Vinculación y Extensión Universitaria DGV YEU}

Esta Dirección tiene una ubicación de primer nivel en la estructura de la UAEM, ella depende directamente de la Rectoría. Tiene el objetivo de gestionar y de concertar convenios con los sectores público, privado y social para cumplir, como reza su misión, «con el propósito de vincularse y trabajar en favor del desarrollo de la ciencia, la investigación y la cultura, tanto en beneficio de la propia comunidad universitaria como de las organizaciones con las cuales se establecen los acuerdos de trabajo conjunto» (Morales 1995: EXT -03).

Según el informe de Rectoría, en 1995 se firmaron 7 convenios con representantes de sectores sociales, productivos y públicos. Se realizaron 14 acuerdos de colaboración, 10 convenios derivados y ningún contrato firmado.

Esta Dirección ha negociado que los organismos empresariales sesionen en el claustro universitario, sin embargo hasta ahora, solamente la vinculación se ha reducido a la firma de convenios. El responsable de la vinculación comenta que hasta ahora no ha sido necesario hacer un contrato de transferencia de tecnología o el trámite de alguna patente (Porcayo 1996:c.p.).

Dentro de sus actividades, la DGV y EU ha editado una serie de catálogos de servicios y capacitación de cada uno de los organismos de la UAEM. En 1996 se publicará un sólo catálogo de servicios y capacitación de la UAEM con un directorio de los organismos a los que puede recurrirse para obtener lo requerido. De la gama de servicios y productos que se ofrecen van desde el fotocopiado, la maquila de envasado de frutas y legumbres hasta la disponibilidad de realizar análisis de alimentos, de microbiología farmacéutica. Pero la desvinculación de la Universidad es notoria ante la ausencia de contratos donde la UAEM brinde algún servicio relacionado con la investigación tecnológica (DGE y VU 1996). Seria prolijo enumerar los servicios que ofrece el conjunto de escuelas y facultades. Sin embargo, es claro que no existe una política del uso de los recursos físicos y humanos de la UAEM. Los funcionarios no tienen claro si los laboratorios universitarios deben ser puestos a funcionar como maquiladores de la industria o si es función de una escuela ofrecer el servicio de revelado de fotografías.

La intensidad de la función de vinculación corresponde con el bajo nivel de intensidad de investigación y con la ausencia de un marco jurídico apropiado y orientador de la innovación tecnológica. Además de lo anterior, parece no haber argumentos sólidos para justificar la existencia de dos dependencias dedicadas a la misma actividad de vinculación. 


\section{Conclusiones}

Después de exponer los diferentes elementos, podemos obtener algunas conclusiones: Respecto al marco jurídico no se perciben elementos que normen la investigación orientada a la innovación tecnológica, ninguno de los documentos declara enfáticamente este interés. Por el contrario se encuentran declaraciones que aluden al avance de la investigación científica como la forma normal del que hacer universitario.

Esta orientación científica de la investigación en las Universidades mexicanas ha sido comentada por diferentes personas e instituciones (López 1995:c.p., Chazaro 1995:374, OECD 1995, etc.). El marco jurídico de la UAEM no permite apoyar claramente y sin restricciones la innovación tecnológica y la investigación aplicada.

En relación a la investigación puede concluirse que esta actividad se realiza de manera dispersa, que explota solamente el $7.6 \%$ del personal académico de la UAEM y el $30 \%$ del personal con posgrado.

La UAEM hace un gran esfuerzo para financiar la investigación con recursos propios, a pesar de esto $3.32 \%$ del total de los proyectos autorizados en 1995 corresponden a la ingeniería y la tecnología.

Hay 7 organismos académicos con cierto grado de experiencia en temas tecnológicos, en tanto que 27 organismos realizan investigación en una muy baja escala. La investigación tecnológica representa entre $12.5 \%$ y $9.9 \%$ del total de los proyectos según los criterios de área de conocimiento del CONACYT o según el tipo tecnológico de los proyectos, correspondientemente.

La vinculación Universidad-Sector productivo es escasa y desordenada. La presencia de dos entidades responsables de esta actividad no coadyuva a la creación del medio ambiente necesario al desarrollo de la investigación en innovación tecnológica. La ubicación institucional de la Dirección General de Vinculación es estratégica y de primer orden, pareciera que su influencia se extiende a toda la UAEM, sin embargo no puede determinarse si la importante ubicación de esta Dirección respecto al organigrama universitaria tiene una función significante debido a las demandas oficiales vinculadas por la ANUIES, la SEP y el CONACYT hacia la función de vinculación.

Bajo este contexto se proponen algunas recomendaciones, que se formulan en dos niveles de perspectiva de aplicación: de un lado la replica de estos ejercicios de estudio de la innovación tecnológica en las universidades públicas mexicanas (y esto comprende a la propia UAEM) y de otro lado, plantear recomendaciones a la UAEM para avanzar hacia una política de innovación tecnológica. 
En relación con la primera recomendación, se considera que las universidades deberían desarrollar una política de estudio y reflexión sobre su actividad de investigación. La UAEM requiere de un Centro de estudios sobre la actividad científico-tecnológica como el instrumento más idóneo para acopiar, procesar y discutir la información sobre la investigación en la UAEM. Este Centro podría, también, desarrollar metodologías para profundizar y avanzar los estudios sobre la investigación y la innovación en la UAEM.

Respecto a la innovación tecnológica se considera imprescindible que la UAEM discuta y acuerde una política sobre la innovación tecnológica y la investigación aplicada. Se considera necesario que los universitarios reflexionen e intercambien opiniones para reformar sobre su acción en la innovación tecnológica. Es importante aclarar que de conformidad con las nociones teóricas utilizadas, la UAEM es un actor de la innovación aunque su papel sea marginal o incluso inactivo; de todas formas, ella no puede marginarse de una cuestión crucial como su participación en la innovación tecnológica. Dicho de otra manera la UAEM puede formar parte de la Red Socio-técnica de la innovación regional en la medida que reflexione y reoriente su actividad de investigación.

La UAEM requiere pensar en la constitución de una red en la que juegue un papel vital. Para lo anterior es necesario que la UAEM reforme su legislación, organice su investigación y establezca una estructura burocrática orientados hacia el avance de la innovación tecnológica. Sólo una reforma original y global de la UAEM puede establecer una Red disponible a la innovación y que le permita explotar en toda su capacidad los recursos con los que cuenta actualmente.

\section{Bibliografía.}

Arellano-Hernández, A., 1996: L'bybridation du mars et des agriculteurs dans les Hautes Vallées du Mexique (La production des objets techniques agricoles). Thèse de doctoral en Anthropologie. Dept d'Anthropolgie. Université Laval. (Inédit).

Arvanitis, R. et al. 1986: Évaluation des politiques publiques de la recherche scientifique et de la technologie, Paris: Mission scientifique et technique.

Barone, F. 1989. «Science et technologie: un rapport entre deux ambiguites $\gg$. en Fundamenta Scientiae. 10.

Basaglia, 1991: La Evolución de la Técnica, México: Crítica.

Bijker, W. E., T. P Hughes, y T.J Pinch, 1993: The Social Construction of Technological Systems, Cambridge: MIT Press. 
Callon, M., 1981: «Por une socioloie des controverses technologiques» en Fundamenta Scientia 2.

Callon, M, 1986: Sociologie de la traduction, L'année sociologique.

Chazaro, L., 1995: "R\&D at the Autonomus National University of Mexico", en Industry \& Higher Education. Decembre: 374-378.

CIC (Coordinación de investigación científica), 1983: Evolución de la investigación en la UAEM, Toluca ( $\mathrm{Mx})$ UAEM.

CGIC (Coordinación General de Investigación Científica), 1990. Informe de actividades del año 1990. Toluca (Mx). UAEM.

DGE y VU (Dirección General de Extensión y Vinculación Universitaria), 1996: Convenios firmados por la UAEM 1993-1995, Toluca (Mx) UAEM.

Esteva, J.A., 1995: "La Universidad frente al reto de la innovación tecnológica: dilemas y oportunidades” en: Compendio sobre la gestión de la innovación tecnológica, México: CIT -UNAM.

Federal Departements and Agencies, 1981: Principes for the evaluation of programs, Otawa: FDA

García- Torres, A. 1990: «Planeación estratégica y planeación tecnológica» en Gestión Tecnológica en la Empresa, Santiago de Chile: BID-SECABCINDA.

Georghiou, L. Gibbons, M., 1987: Evaluation of applied research, s/ed. Hernández, A., 1996: Comunicación personal.

Hughes, T., 1983: «L'électrification de l'Amérique» en Culture technique.

Latour, B., 1986: «L objet des sciences, raison d être des approches sociosemiotiques, les discours du savoir. Cahiers de I ACFAS du Québec. ACFAS.

Law, J., 1984: «Sur la tectique du control social».Cahiers du STS:106-126.

Leroi-Gourhan, A., 1945: Le geste et la parole. I techniques et langage, Paris: Albin Michel.

Lomnitz, A., M.D. Leon, y C.R. Díaz, 1988: "Del laboratorio universitario al taller industrial”. En Ciencia y Desarrollo. No 80, año XIV, Mayo/junio: $41-54$.

López, R. Y J.L. Solleiro R., 1995: "La experiencia reciente de vinculación universidad-empresa en México" en: Compendio sobre gestión de la innovación tecnológica, México. CIT -UNAM

Mauss, M. 1927: Manuel d'ethnographie, Paris: Payot.

Morales, G. M. A., 1995: Tercer informe de actividades (del rectorado 19931997), Toluca, UAEM. 
OECD, 1981: La mesure des activités scientifiques et techniques, Paris: OECD.

OECD, 1995: Informe sobre Mexico. s/p. s/e.

Perrin, 1987: Comment naissent les techniques. la production sociales des techniques, Paris. Publisud.

Pinch, T., 1985: «Observer la nature ou observer les instruments» Culture technique. No 14.

Porcayo, G., 1996: Comunicación personal.

PNUD, 1991: Taller sur-sur sobre vinculación Universidad-Empresa, Méx. PNUD.

PRDC y T, s/a: Esquema de los análisis de los recursos humanos cientifico-tecnológicos.

Simondon, G., 1989: Le mode d'existence des objets techniques, Paris: Aubier. Smith, K. 1992: «Technological innovation indicators: experience and prospects» en Innovation. No 9, Vol. 6, Dec.

UNAM, 1985: Proyectos Orientados a la Producción de Bienes y Servicios, México. UNAM.

Victorino, R. L., 1995: "La investigación en la UACh". Convergencia. Año 3, Nos 8/9. Sept, Toluca, UAEM.

Viladiu, M., L. Escribano, L. Bellavista, M. Grabulós, E. Guardiola, C. Iglesias, and D. Serrat, 1992: "A research evaluation model of a large ancient university” en Research Evaluation. Vol. 2, No 3:124-134.

Waissbluth, M., G. Cadena, y J. L. Solleiro, 1988: "La vinculación Universidad-Industria Una experiencia organizacional en México” en: Cuaderno 4, Investigaciones del Centro para la innovación tecnológica, México: CIT -UNAM. 


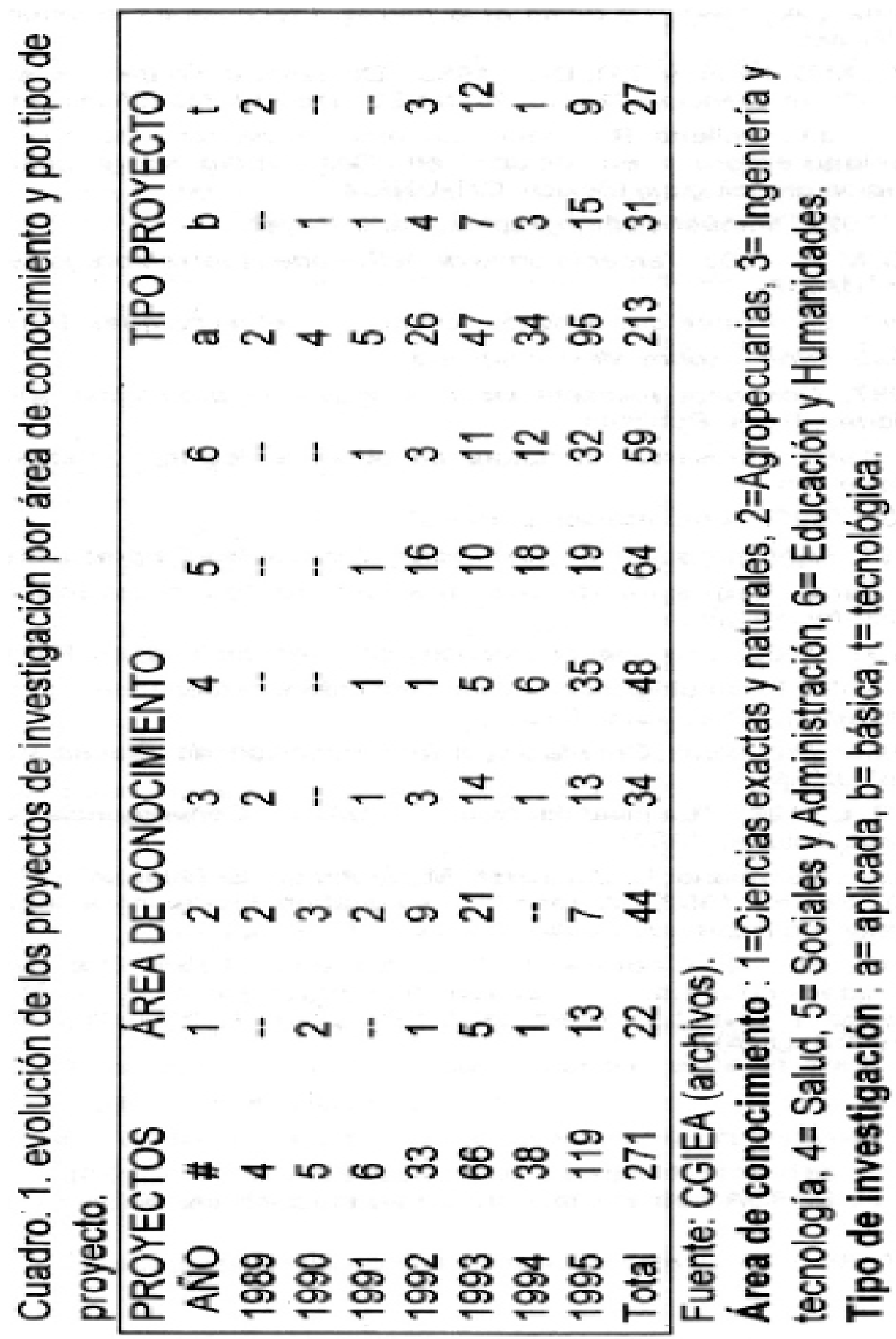


Convergencia Revista de Ciencias Sociales, núm. 12/13, 1996, Universidad Autónoma del Estado de México

웡
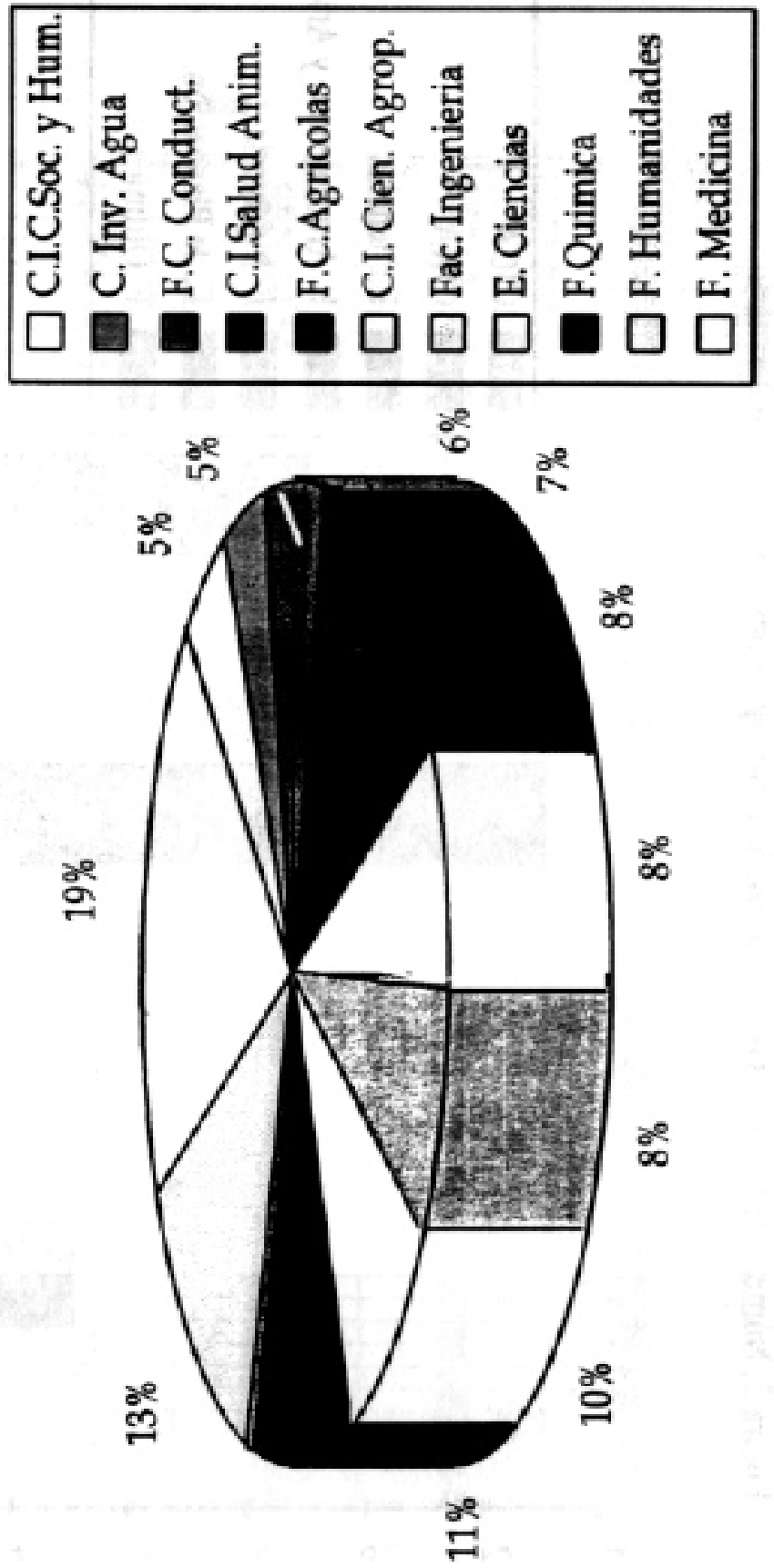


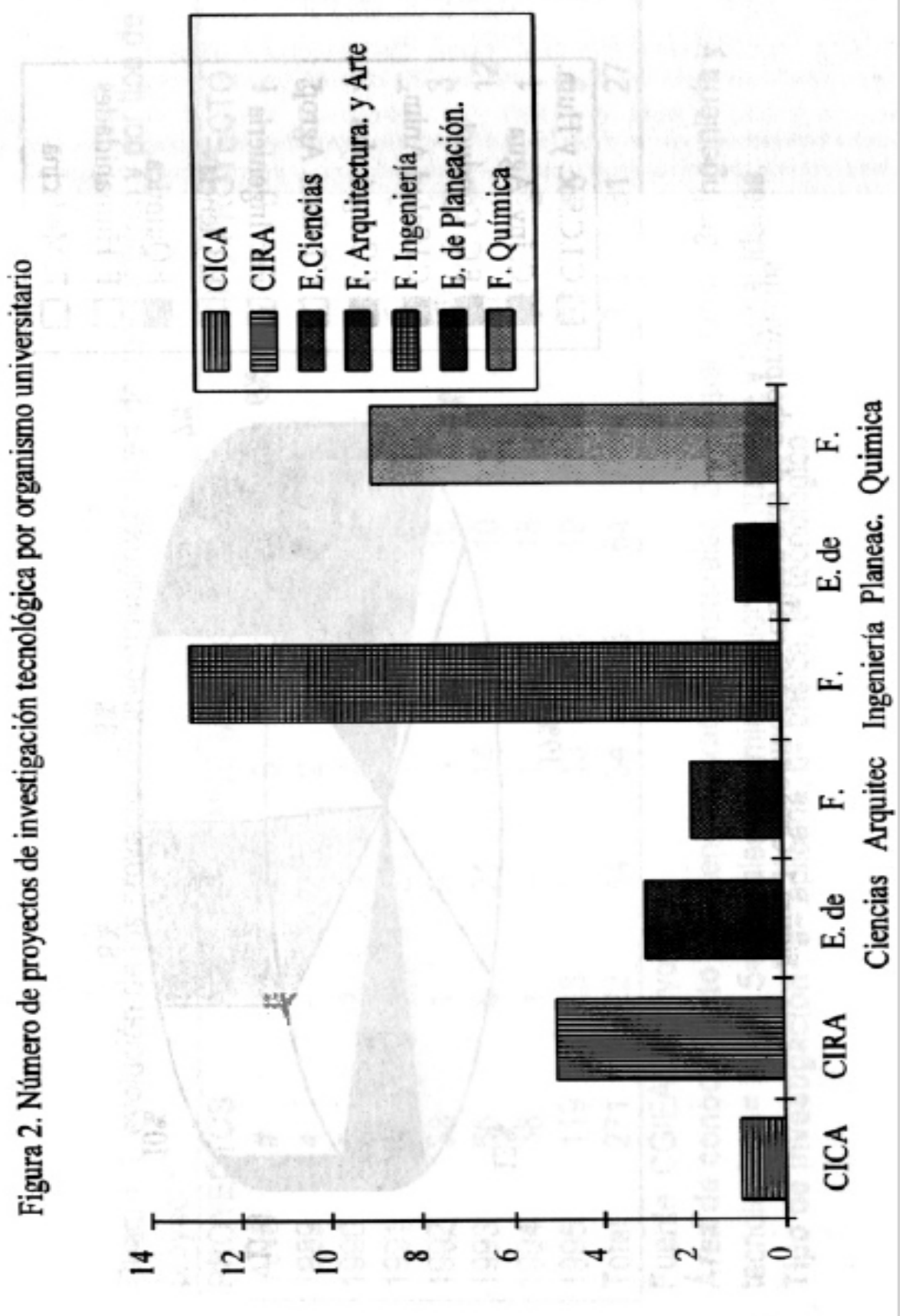


Convergencia Revista de Ciencias Sociales, núm. 12/13, 1996, Universidad Autónoma del Estado de

México

ANEXO 1

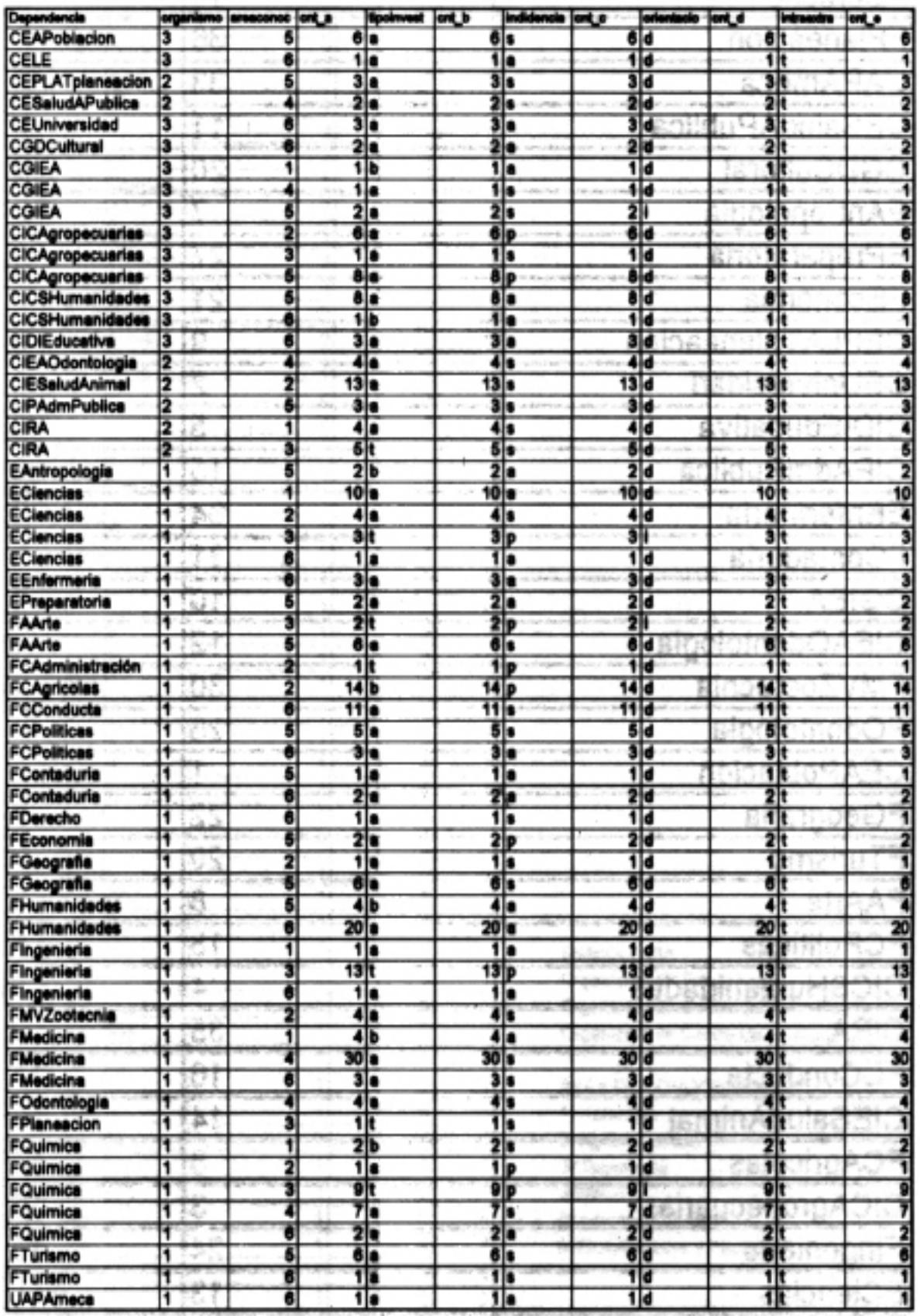




\section{ANEXO 2}

\begin{tabular}{|c|c|c|}
\hline Dependencia & nodeptnden & Ent \\
\hline CELE & $\begin{array}{r}12 \\
\end{array}$ & 1 \\
\hline FCAdminisiracion & 17 & 1 \\
\hline FDemcho & 19 & 1 \\
\hline FPlaneacian & 36 & 1 \\
\hline UAPAmeca & 33 & 1 \\
\hline CESsludAPublica & 111 & 2 \\
\hline CODCuliural & 20 & 2 \\
\hline EAntropologin & 7 & 2 \\
\hline EPreparatoria & 27 & 2 \\
\hline FEconomia & 21 & 2 \\
\hline CEPLAT planeagon & 9 & 3 \\
\hline CEUniversidad & 2 & 3 \\
\hline CIDIFducativa & 5 & ] \\
\hline CiPAdmPublica & 15 & 3 \\
\hline EEnfemeria & 34 & 3 \\
\hline FConteduria & 31 & 3 \\
\hline CGIEA & 10 & 4 \\
\hline CIEAOdoniologia & 12 & 4 \\
\hline FMVRootecnia & 30 & 4 \\
\hline FOdeniologia & 26 & 4 \\
\hline CEAPoblacion & 1 & 6 \\
\hline Fopografia & 22 & 7 \\
\hline FTLRSImo & 29 & 7 \\
\hline FAArte & 8 & 8 \\
\hline FCPoliticas & 18 & B \\
\hline CicsHumanidados & 4 & $\overline{9}$ \\
\hline CIRA & 35 & $\theta$ \\
\hline FCConducta & 16 & 11 \\
\hline CIESsludAnimal & 14 & 13 \\
\hline FCAarloolas & B & 14 \\
\hline CICAgropocua rass & 3 & 15 \\
\hline Fingenieria & 24 & 15 \\
\hline EClencias & 13 & 1 16 \\
\hline FQuimica & 20 & 21 \\
\hline FHumanidades & 23 & 24 \\
\hline FModicina & 25 & 37 \\
\hline
\end{tabular}


Convergencia Revista de Ciencias Sociales, núm. 12/13, 1996, Universidad Autónoma del Estado de México

\begin{tabular}{|c|c|c|c|}
\hline Organismo & No.Organismo & areaconoc & conta \\
\hline CEAPoblacion & 3 & 5 & 6 \\
\hline CELE & 3 & 6 & 1 \\
\hline CEPLATplaneacion & 2 & 5 & 3 \\
\hline CESaludAPublica & 2 & 4 & 2 \\
\hline CEUniversidad & 3 & 6 & 3 \\
\hline CGDCultural & 3 & 6 & 2 \\
\hline CGIEA & 3 & 4 & 1 \\
\hline CGIEA & 3 & 5 & 2 \\
\hline CICApropecuarias & 3 & 2 & 5 \\
\hline CICAgropecuarias & 3 & 3 & 1 \\
\hline CICAgropecuarias & 3 & 5 & 8 \\
\hline CICSHumanidades & 3 & 5 & 7 \\
\hline CIDIEducativa & 3 & 6 & 3 \\
\hline CIEAOdontologia & 2 & 4 & 4 \\
\hline CIESaludAnimal & 2 & 2 & 13 \\
\hline CIPAdmPublica & 2 & 5 & 3 \\
\hline CIRA & 2 & 1 & 4 \\
\hline CIRA & 2 & 3 & 1 \\
\hline ECiencias & 1 & 1 & 10 \\
\hline ECiencias & 1 & 2 & 4 \\
\hline ECiencias & 1 & 6 & 1 \\
\hline EEnfermeria & 1 & 6 & 3 \\
\hline EPreparatoria & 1 & 5 & 2 \\
\hline FAArte & 1 & 3 & 1 \\
\hline FAArte & 1 & 5 & 6 \\
\hline FCAgricolas & 1 & 2 & 9 \\
\hline FCConducta & 1 & 6 & 11 \\
\hline FCPoliticas & 1 & 5 & 4 \\
\hline FCPoliticas & 1 & 6 & 3 \\
\hline FContaduria & 1 & 5 & 1 \\
\hline FContaduria & 1 & 6 & 2 \\
\hline FDerecho & 1 & 6 & 1 \\
\hline FEconomia & 1 & 5 & 2 \\
\hline FGeografia & 1 & 2 & 1 \\
\hline FGeografia & 1 & 5 & 6 \\
\hline FHumanidades & 1 & 5 & 3 \\
\hline FHumanidades & 1 & 6 & 7 \\
\hline Fingenieria & 1 & 1 & 1 \\
\hline Fingenieria & 1 & 3 & 1 \\
\hline Fingenieria & 1 & 6 & 1 \\
\hline FMVZootecnia & 1 & 2 & 4 \\
\hline FMedicina & 1 & 1 & 1 \\
\hline FMedicina & 1 & 4 & 29 \\
\hline FMedicina & 1 & 6 & 3 \\
\hline FOdontologia & 1 & 4 & 4 \\
\hline FQuimica & 1 & 1 & 1 \\
\hline FQuimica & 1 & 2 & 1 \\
\hline FQuimica & 1 & 3 & 4 \\
\hline FQuimica & 1 & 4 & 7 \\
\hline FQuimica & 1 & 6 & 2 \\
\hline FTurismo & 1 & 5 & 6 \\
\hline FTurismo & 1 & 6 & 1 \\
\hline UAPAmecA & 1 & 6] & 1 \\
\hline
\end{tabular}

ANEXO 3

SIMBOLOGIA

Organismo 1= Esc. o Fac

Organismo 2 $=$ Centro de Esc. of Fac.

Organismo $3=$ Centro Universitario

AREA DE CONOCIMIENTO

$1=$ Naturales

$2=$ Agropecuarias

3=Tecnologia

$4=$ Salud

$5=$ Admón. $y$ Sociales

6=Humanidades y Educación 
Antonio Arellano Hernández . La Capacidad de Innovación Tecnológica en la Universidad Autónoma del Estado de México

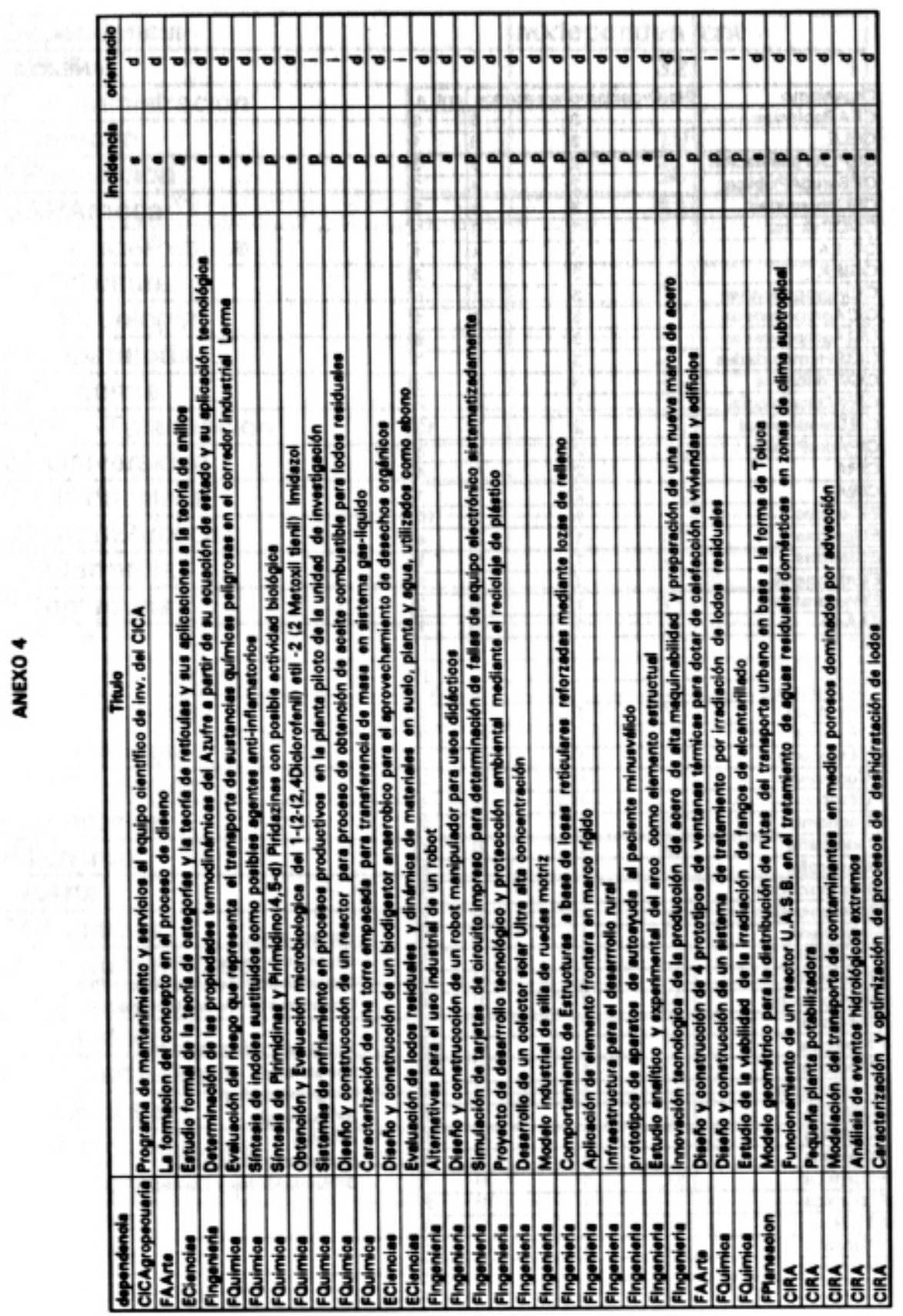


Convergencia Revista de Ciencias Sociales, núm. 12/13, 1996, Universidad Autónoma del Estado de México

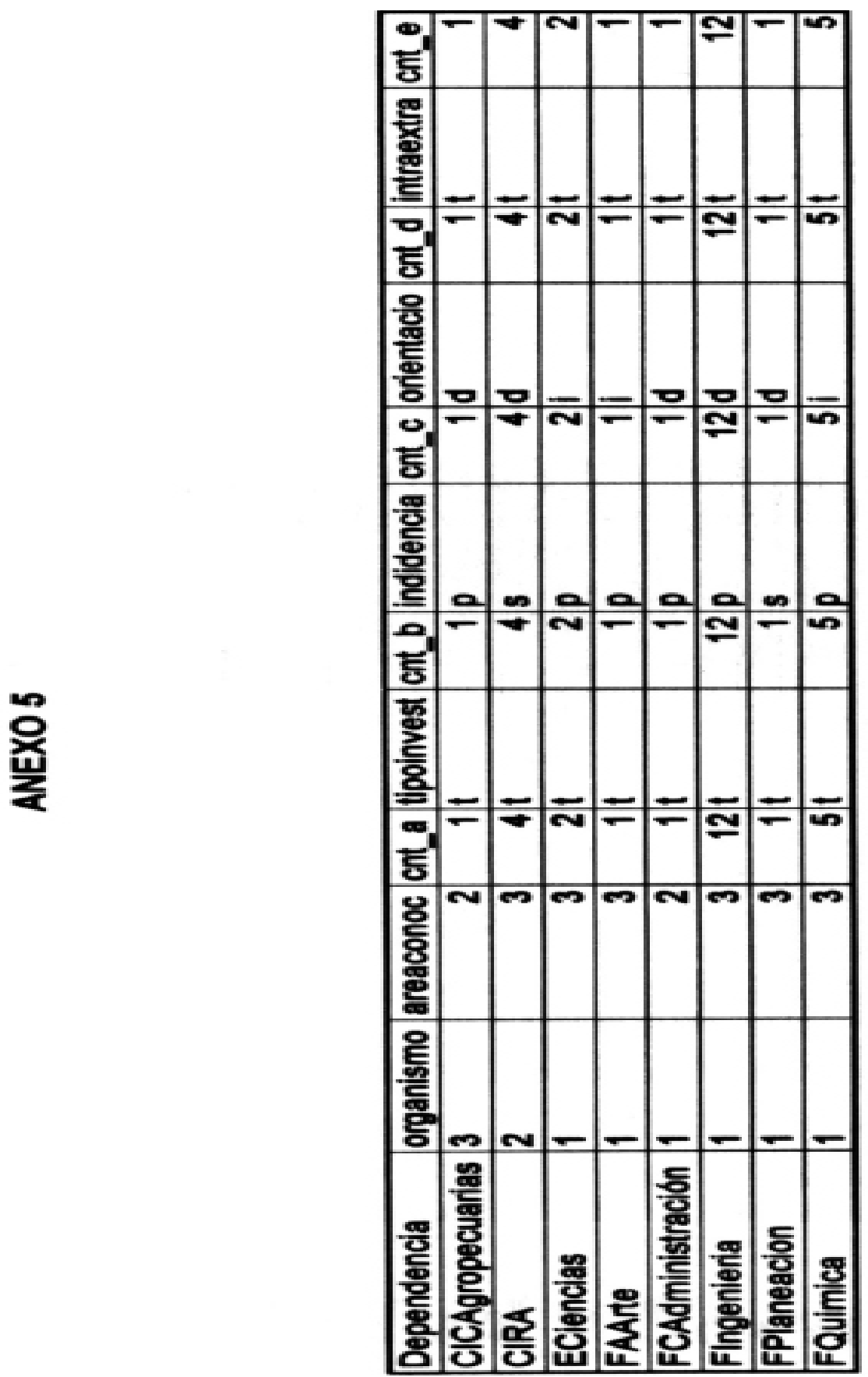

